\title{
La coadministración de la vacuna COVID-19 con la de la influenza estacional demostró ser segura, inmunogénica y eficaz
}

Co-administration of the COVID-19 vaccine with that of seasonal influenza demonstrated to be safe, immunogenic and effective

\section{Comentado de:}

Toback S, et al. medRxiv 2021:2021.2006.2009.21258556 ${ }^{1}$

\section{Antecedentes}

Hasta el momento era desconocido el perfil de seguridad e inmunogenicidad de las vacunas COVID-19 administradas de manera concomitante con vacunas contra la influenza estacional.

\section{Métodos}

Subestudio de coadministración de la vacuna contra la influenza del ensayo aleatorizado fase 3 sobre seguridad y eficacia de la vacuna contra COVID-19 NVX-CoV2373 (Novavax ${ }^{\circledR}$ ), que utiliza nanopartículas de proteínas recombinantes y matrix-M como adyuvante $^{2}$. A los primeros participantes que cumplieron con los criterios de ingreso al ensayo y que no tenían contraindicaciones para la vacunación contra la influenza se los invitó a participar del subestudio, aleatorizándolos en forma estratificada por sitio y por edad (mayores o menores de 65 años), a recibir NVX-CoV2373 $(n=217)$ o placebo $(n=214)$, con 21 días de diferencia. Los 431 participantes recibieron, de manera abierta, una única inyección intramuscular $(0,5 \mathrm{~mL})$ de una vacuna antigripal autorizada en el deltoides opuesto (tetravalente en los participantes de 18 a 64 años y trivalente en los participantes mayores de 65 años) sólo junto a la primera aplicación de la vacuna del ensayo.

La reactogenicidad fue evaluada a través de un diario electrónico durante los siete días posteriores a la vacunación, así como los eventos adversos (EA) que requirieron atención médica
(EAAM) y los serios (EAS). Fueron realizados ensayos de inhibición de la hemaglutinación de la influenza y de IgG anti-espiga de SARS-CoV-2. Fue evaluada la eficacia de la vacuna contra COVID-19 sintomática confirmada por PCR. Se hicieron comparaciones entre los participantes del subestudio y del estudio principal.

\section{Resultados}

Los participantes del subestudio fueron más jóvenes, de mayor diversidad racial y con menos comorbilidades que los participantes del estudio principal. Los eventos de reactogenicidad más comunes en el grupo de coadministración vs. dosis única incluyeron pequeñas diferencias en el desarrollo de sensibilidad $(70,1$ vs. $57,6 \%)$ o dolor (39,7 vs. $29,3 \%$ ) en el lugar de la inyección, fatiga ( 27,7 vs. $19,4 \%$ ) y dolor muscular ( 28,3 vs. $21,4 \%)$.

Las tasas de EA, EAAM y EAS fueron bajas y equilibradas entre los dos grupos. La coadministración no produjo cambios en la respuesta inmune de la vacuna contra la influenza, observándose una reducción en las respuestas de anticuerpos a la vacuna NVX-CoV2373. La eficacia de la vacuna para casos confirmados de la variante británica B.1.1.7 fue de $87,5 \%$ en el subestudio y de $89,8 \%$ en el estudio principal.

\section{Conclusión}

La coadministración de la vacuna COVID-19 con la de la influenza estacional demostró ser segura, inmunogénica y eficaz.

\section{Comentario}

Los enormes esfuerzos mundiales de vacunación contra el SARS-CoV-2, con más de 2.412 millones de dosis de vacunas administradas al 20 de junio de $2021^{3}$, no tienen precedentes. Este programa continuo de vacunación masiva contra la COVID-19 tiene coincidencias estacionales con los programas de vacunación contra la influenza. Es en este contexto que la interferencia inmunológica y la seguridad son una preocupación cuando dos vacunas son administradas en forma simultánea. Los autores buscaron en PubMed estudios publicados desde diciembre de 2019 hasta el 1 de abril de 2021 sin restricciones de idioma para los términos "SARS-CoV-2", ÇOVID-19", "vacuna", çoadministración. einmunogenicidad". No identificaron publicaciones revisadas por pares que describieran el uso simultáneo de cualquier vacuna contra el SARS-CoV-2 y otra vacuna. Por otro lado, quien firma este comentario actualizó la búsqueda el 21 de Junio de 2021 en la misma base de datos, obteniendo el mismo resultado. Además, los ensayos de las vacunas COVID-19 tenían, con frecuencia, criterios de que excluían específicamente a aquellos con vacunación -reciente o planificada- con cualquier vacuna autorizada, en una fecha cercana o en el momento de cualquier inyección del estudio.

Por dicha razón y ante la falta de evidencia, en el mundo se han venido recomendando intervalos libres de otras vacunas antes y después de la vacuna COVID-19 (siete días en el Reino Unido ${ }^{4}$ y 14 días en el caso de Argentina ${ }^{5}$ y EE.UU. ${ }^{6}$ ), pero priorizando esta última. Si bien esta estrategia conservadora puede evitar complicaciones, por otro lado genera dudas en la población, que no tiene certeza de turnos, y en muchos casos, pérdidas de oportunidades de vacunación contra el SARS-CoV-2 en momentos críticos en la lucha contra la pandemia. La coadministración, en cambio, podría aumentar la tasa de cobertura de ambas vacunas.

Este es el primer estudio que demuestra un adecuado perfil de seguridad, inmunogenicidad y eficacia de una vacuna COVID19 cuando es coadministrada con vacunas contra la influenza estacional. Las limitaciones del estudio, además de no haber sido aún revisado por pares (prepublicación), incluyen su pequeño tamaño muestral (hubo pocos participantes con más de 65 años de edad debido a la alta tasa de vacunación antigripal en forma rutinaria de este colectivo), un pequeño número de desenlaces de eficacia del subestudio, la falta de la evaluación estadística preespecificada de no inferioridad de la inmunogenicidad, la falta de aleatorización en el reclutamiento del subestudio y la administración abierta de la vacuna antigripal. Un 
diseño más sólido habría aleatorizado participantes en cuatro ramas (NVX-CoV2373+ antigripal, NVX-CoV2373+placebo, antigripal+placebo y placebo+placebo). Aunque no se establecieron criterios preespecificados de valoración comparativa de la inmunogenicidad, no se encontraron evidencias de interferencias significativas entre las vacunas. Sólo hubo una modesta reducción de lgG anti-espiga con la coadministración de vacunas. No está claro si esta reducción se debió a la interferencia entre vacunas o a la naturaleza no aleatorizada de los grupos estudiados. Dado que no se correlacionó con pérdida de la protección, es difícil interpretar la importancia de este hallazgo aunque debe considerarse que el pequeño número de casos confirmados en el subestudio hacen imprecisa esta valoración de la eficacia. La reducción de anticuerpos anti-espiga puede ser menos relevante en los participantes que son seropositivos al inicio del estudio, ya que ellos alcanzaron valores $59 \%$ más altos con la coadministración en comparación con los receptores de CoV2373 sola. Por lo tanto, es posible que la vacuna contra la influenza no tenga ese impacto cuando es coadministrada con la segunda dosis de refuerzo de la vacuna COVID-19.

\section{Conclusiones del comentador}

Este estudio proporciona información clave para ayudar a guiar la toma de decisiones de políticas nacionales de inmunización sobre el uso concomitante de vacunas COVID-19 y antigripales. Los resultados sugieren que la vacunación concomitante puede ser una estrategia de inmunización viable, especialmente para la segunda dosis de vacunas COVID-19. Probablemente también lo sea para la primera dosis, pero dado el escaso número de casos COVID-19 registrados en el subestudio, podría ser razonable sugerir un intervalo con la primer dosis de una vacuna COVID-19, aunque ante una accesibilidad limitada y en el contexto de un pico pandémico, ninguna vacuna COVID-19 debería ser suspendida.

Agustin Ciapponi [ Servicio de Medicina Familiar y Comunitaria, Hospital Italiano de Buenos Aires; Centro Cochrane Argentina, Instituto de Efectividad Clínica y Sanitaria; Centro Cochrane Argentina, Instituto de Efectividad Clínica y Sanitaria. aciapponi@gmail.com ]

Ciapponi A La coadministración de la vacuna COVID-19 con la de la influenza estacional demostró ser segura, inmunogénica y eficaz. Evid Actual Pract Ambul. 2021;24(2):e002137. Available from: https://dx.doi.org/10.51987/EVIDENCIA.V24I3.6953. Comentado de: Toback S et al. medRxiv 2021:2021.2006.2009.21258556

\section{Referencias}

1. Toback S, Galiza E, Cosgrove C, et al. Safety, Immunogenicity, and Efficacy of a COVID-19 Vaccine (NVX-CoV2373) Co-administered With Seasonal Influenza Vaccines. medRxiv. 2021;Available from: 10.1101/2021.06.09.21258556.

2. A Study Looking at the Effectiveness, Immune Response, and Safety of a COVID-19 Vaccine in Adults in the United Kingdom;. Available from: https://ClinicalTrials.gov/show/NCT04583995;2021.

3. World Health Organization Coronavirus (COVID-19) Dashboard; 2021. Available from: https://covid19.who.int/.

4. Public Health England. Annual flu programme. Seasonal influenza (flu) is an unpredictable but recurring pressure that the NHS faces every winter. Vaccination offers the best protection. Updated 14 May 2021.; 2013. Available from: https://www.gov.uk/government/collections/annual-fluprogramme [Last access: 2021-06-21].

5. ¿Primero gripe o coronavirus?: cómo debe ser el orden y las prioridades de vacunación contra enfermedades respiratorias; 2021. Available from: https://www.telam.com.ar/notas/202105/552696-orden-de-vacucacion-contra-enfermedades-respiratorias.html.

6. Centers for Disease Control and Prevention. Interim clinical considerations for use of COVID-19 vaccines currently authorized in the United States; 2021. Available from: https://www.cdc.gov/vaccines/covid-19/clinical-considerations/covid-19-vaccines-us.html [Last access: 2021-06-22]. 